\title{
Analysis of Regolith Simulant Ejecta Distributions from Normal Incident Hypervelocity Impact
}

\author{
David L. Edwards ${ }^{1}$, William Cooke ${ }^{2}$, Rob Suggs ${ }^{3}$ \\ National Aeronautics and Space Administration/George C. Marshall Space Flight Center, \\ M/S EV44 MSFC, AL 35812 USA \\ Danielle E. Moser ${ }^{4}$ \\ Stanley/MSFC, AL 35812 USA
}

The National Aeronautics and Space Administration (NASA) has established the Constellation Program. The Constellation Program has defined one of its many goals as long-term lunar habitation. Critical to the design of a lunar habitat is an understanding of the lunar surface environment; of specific importance is the primary meteoroid and subsequent ejecta environment. The document, NASA SP-8013 "Meteoroid Environment Model Near Earth to Lunar Surface", was developed for the Apollo program in 1969 and contains the latest definition of the lunar ejecta environment. There is concern that NASA SP-8013 may over-estimate the lunar ejecta environment. NASA's Meteoroid Environment Office (MEO) has initiated several tasks to improve the accuracy of our understanding of the lunar surface ejecta environment.

This paper reports the results of experiments on projectile impact into powdered pumice and unconsolidated JSC-1A Lunar Mare Regolith simulant targets. Projectiles were accelerated to velocities between 2.45 and $5.18 \mathrm{~km} / \mathrm{s}$ at normal incidence using the Ames Vertical Gun Range (AVGR). The ejected particles were detected by thin aluminum foil targets strategically placed around the impact site and angular ejecta distributions were determined. Assumptions were made to support the analysis which include; assuming ejecta spherical symmetry resulting from normal impact and all ejecta particles were of mean target particle size. This analysis produces a hemispherical flux density distribution of ejecta with sufficient velocity to penetrate the aluminum foil detectors.

\footnotetext{
${ }^{1}$ Branch Chief, Natural Environments Branch,EV44

${ }^{2}$ Lead, Meteoroid Environments Office, EV44

${ }^{3}$ Team Lead, Space Environment Team, EV44

${ }^{4}$ Engineer, Stanley \& Associates, EV44
} 


\title{
Analysis of Regolith Simulant Ejecta Distributions from Normal Incident Hypervelocity Impact
}

\author{
David L. Edwards ${ }^{1}$, William Cooke ${ }^{2}$, Rob Suggs ${ }^{3}$ \\ National Aeronautics and Space Administration/George C. Marshall Space Flight Center, \\ $M / S$ EV44 MSFC, AL 35812 USA \\ Danielle E. Moser ${ }^{4}$ \\ Stanley/MSFC, AL 35812 USA
}

The National Aeronautics and Space Administration (NASA) has established the Constellation Program. The Constellation Program has defined one of its many goals as long-term lunar habitation. Critical to the design of a lunar habitat is an understanding of the lunar surface environment; of specific importance is the primary meteoroid and subsequent ejecta environment. The document, NASA SP-8013 "Meteoroid Environment Model Near Earth to Lunar Surface", was developed for the Apollo program in 1969 and contains the latest definition of the lunar ejecta environment. There is concern that NASA SP-8013 may over-estimate the lunar ejecta environment. NASA's Meteoroid Environment Office (MEO) has initiated several tasks to improve the accuracy of our understanding of the lunar surface ejecta environment.

This paper reports the results of experiments on projectile impact into powdered pumice and unconsolidated JSC-1A Lunar Mare Regolith simulant targets. Projectiles were accelerated to velocities between 2.45 and $5.18 \mathrm{~km} / \mathrm{s}$ at normal incidence using the Ames Vertical Gun Range (AVGR). The ejected particles were detected by thin aluminum foil targets strategically placed around the impact site and angular ejecta distributions were determined. Assumptions were made to support the analysis which include; assuming ejecta spherical symmetry resulting from normal impact and all ejecta particles were of mean target particle size. This analysis produces a hemispherical flux density distribution of ejecta with sufficient velocity to penetrate the aluminum foil detectors.

\section{INTRODUCTION}

The series of tests discussed in this paper grew out of two focus areas, related to exploration of the lunar surface. The primary goal of this series of tests was to determine the fraction of impact energy which is converted to visible light. The other focus was the need to increase our understanding of the ejecta environment on the lunar surface. NASA's Meteoroid Environment Office (MEO) offered the opportunity to gather ejecta distribution data during a series of test shots using the Ames Vertical Gun Range (AVGR).

The AVGR is a 0.30 caliber light gas gun that can launch projectiles to velocities ranging from $0.5 \mathrm{~km} / \mathrm{s}$ to nearly $7 \mathrm{~km} / \mathrm{s}$. A very unique feature of the AVGR is the ability to vary the gun's angle of elevation with respect to the target. The angle of elevation of the gun can be varied in 15-degree increments from 0 to 90 degrees, thus permitting oblique angles of impact. Impact events can be recorded with a variety of high-speed imaging options ${ }^{1}$.

\footnotetext{
1 Branch Chief, Natural Environments Branch,EV44

${ }^{2}$ Lead, Meteoroid Environments Office, EV44

${ }^{3}$ Team Lead, Space Environment Team, EV44

${ }^{4}$ Engineer, Stanley \& Associates, EV44
} 
NASA SP-8013 describes the ejecta environment subsequent to meteoroid impact ${ }^{2}$. The graph shown in Fig. 1 indicates approximately a 4 order of magnitude increase in the number of ejecta particles of mass, $m$, from the baseline primary meteoroid impact flux of mass, $m$, as defined by the Grü model ${ }^{3}$. The design of a long-term habitation structure to survive the ejecta environment described in NASA SP-8013 would require significant shielding mass, making it difficult and potentially cost prohibitive to launch and deliver the structure to the lunar surface. A better understanding of the lunar ejecta environment is required to optimize the lunar habitat design.

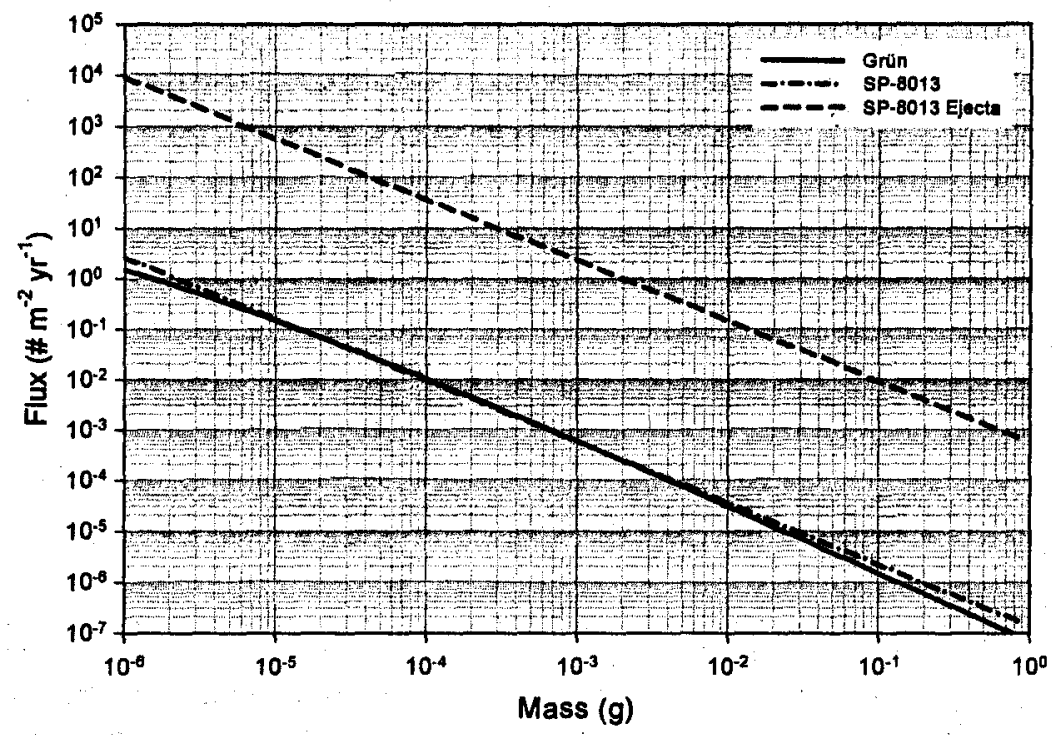

Figure 1. Estimation of the lunar ejecta flux compared to the prediction of primary impact flux from the Grün model.

\section{EXPERIMENT}

The purpose of this series of experiments was to collect information enabling the characterization of the ejecta angular distribution resulting from a hypervelocity impact into simulated regolith. These ejecta characterization experiments were secondary experiments in the AVGR, and as such were dependent upon the test parameters required by the primary experiment: the determination of the fraction of impact energy which is converted to visible light. Therefore one of the constraints of the ejecta characterization experiment was that the ejecta experiment could not influence, bias, perturb, or otherwise contaminate the data obtained from the primary experiment. The ejected particles were detected by thin, $0.17 \mathrm{~mm}$, aluminum foil targets placed around the powered targets in a vacuum chamber maintained at a vacuum level of 0.5 Torr during the impact test. The pumice was fine grain, $60 \mu \mathrm{m}$ diameter particles and the JSC-1A was a larger grain powdered target with mean grain size of $188 \mu \mathrm{m}$ and a median grain size of $103 \mu \mathrm{m}^{4}$

The experiment set-up, shown in Figure 2, consisted of placing sheets of aluminum foil in three specific locations, identified as position " $D$ ", " $B$ ", and " $C$ " around the periphery of the AVGR vacuum chamber. Foil positions " $D$ " and " $B$ " were used to collect ejecta data from the pumice impact test series. Foil position "C" was used to collect ejecta data from the JSC-1 A impact test series. The $0.17 \mathrm{~mm}$ thick foils were positioned such that the ejecta would impact the foils at nearnormal incident angles. The regolith simulants used in this experiment were powdered pumice and JSC-1A. In each case, the projectile was fired vertically $\left(90^{\circ}\right)$ into each powdered regolith simulant." The projectile in each case was $6.35 \mathrm{~mm}$ diameter, $0.29 \mathrm{~g}$ Pyrex sphere. The incident projectile velocities ranged from $2.45 \mathrm{~km} / \mathrm{s}$ to $5.18 \mathrm{~km} / \mathrm{s}$. 


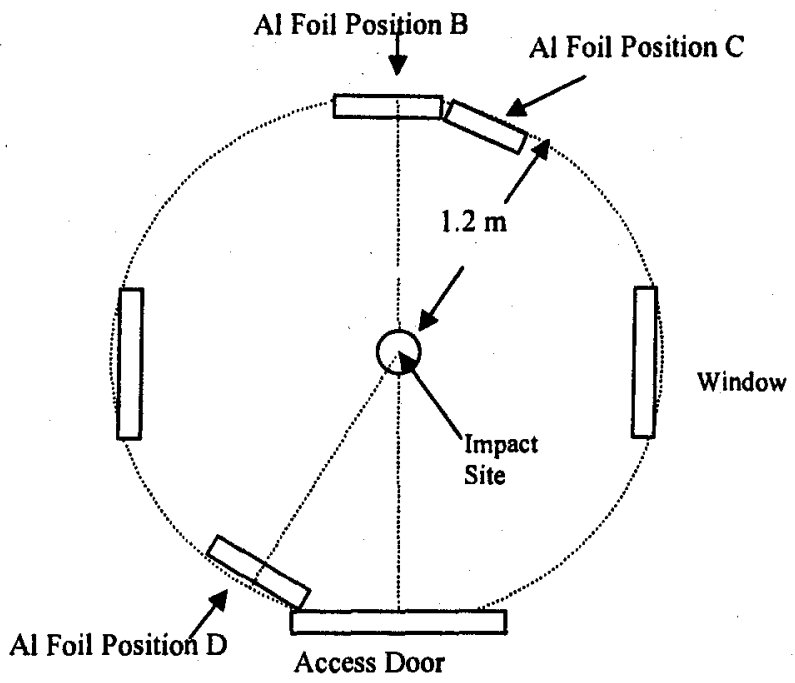

Figure 2. Top view schematic of the test chamber - specifically indicating the positions of the aluminum foil detectors $\mathrm{D}, \mathrm{B}$, and $\mathrm{C}$.

\section{RESULTS}

As shown in Fig 3, the foil targets were divided into $2.54 \mathrm{~cm}$ wide horizontal bands. This bandwidth was approximately equivalent to $1^{\circ}$ as referenced to the impact site. The number of penetrations in each band was counted after the impact test. The number of penetrations was documented as penetrations per unit area. The unit area is the area defined by the $2.54 \mathrm{~cm}$ wide, $30.48 \mathrm{~cm}$ long band. A unit area is defined as $77.42 \mathrm{~cm}^{2}$.
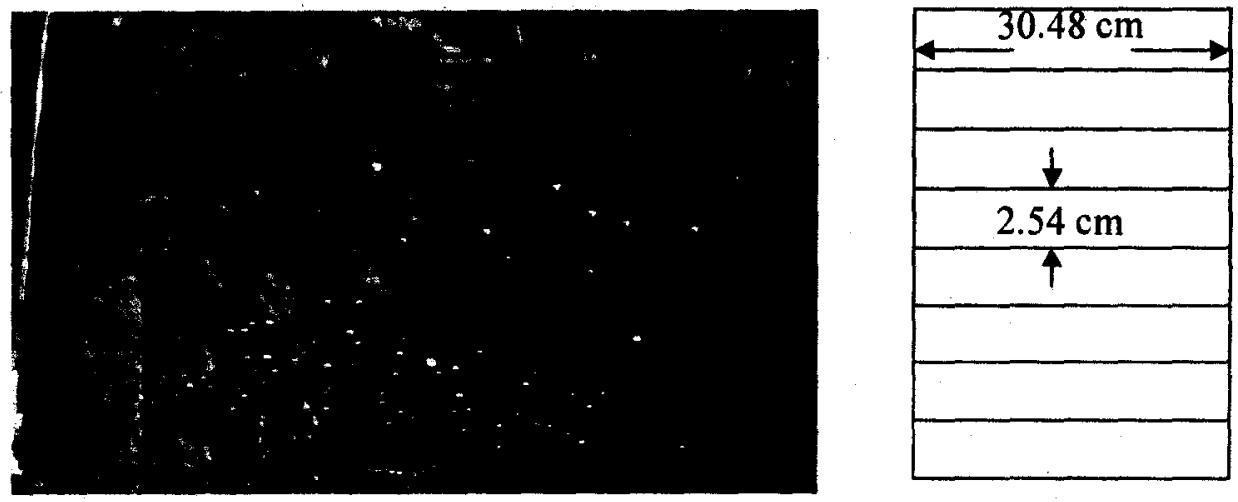

Figure 3. Photograph of an impacted foil and schematic showing how the foils were divided into $1^{\circ}$ bands, $30.48 \mathrm{~cm}$ wide

The JSC-1A targets produced more ejecta penetrations than the pumice target given equivalent incident projectile mass, composition, and velocity. The impact test using powdered pumice performed at $2.5 \mathrm{~km} / \mathrm{s}$ did not produce ejecta that penetrated the foil detectors. The impact test using JSC-1A performed at $2.45 \mathrm{~km} / \mathrm{s}$ produced a significant number of ejecta penetrations. Projectile impact velocities of 3.78 and $5.18 \mathrm{~km} / \mathrm{s}$, into pumice and JSC-1A, produced ejecta that penetrated the foil targets. 
The results from counting penetration in foils are shown in Figures 4 and 5 and indicate a preferred ejecta angle ranging between $38^{\circ}$ to $43^{\circ}$ with respect to the horizontal.

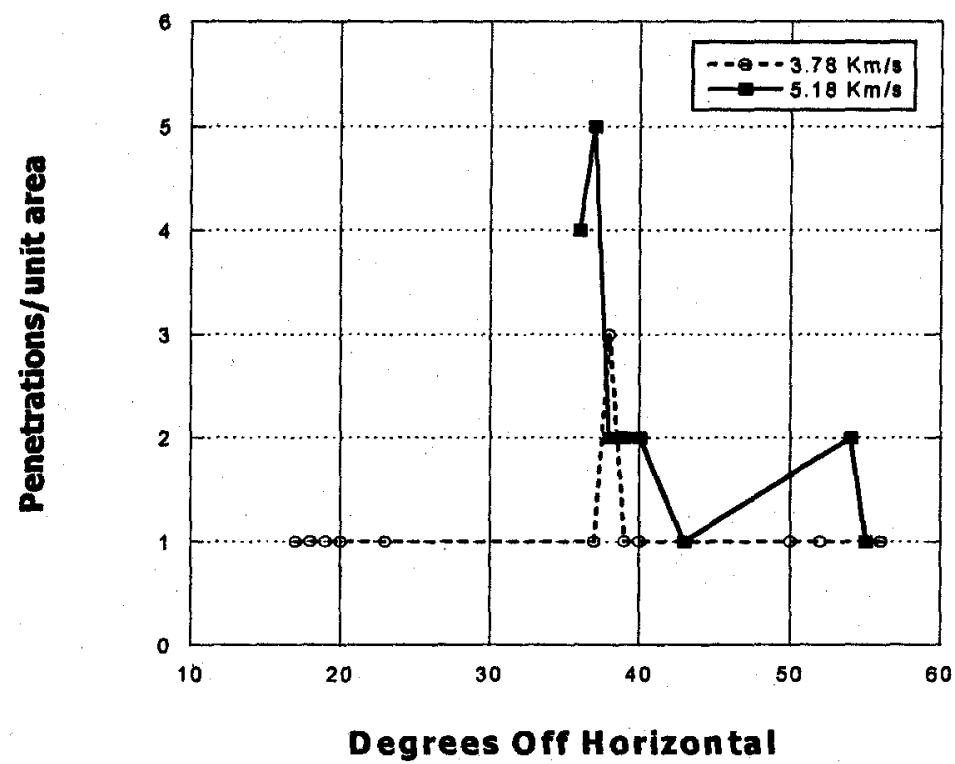

Figure 4. Angular distributions of pumice ejecta with sufficient velocity to penetrate the aluminum foil detectors.

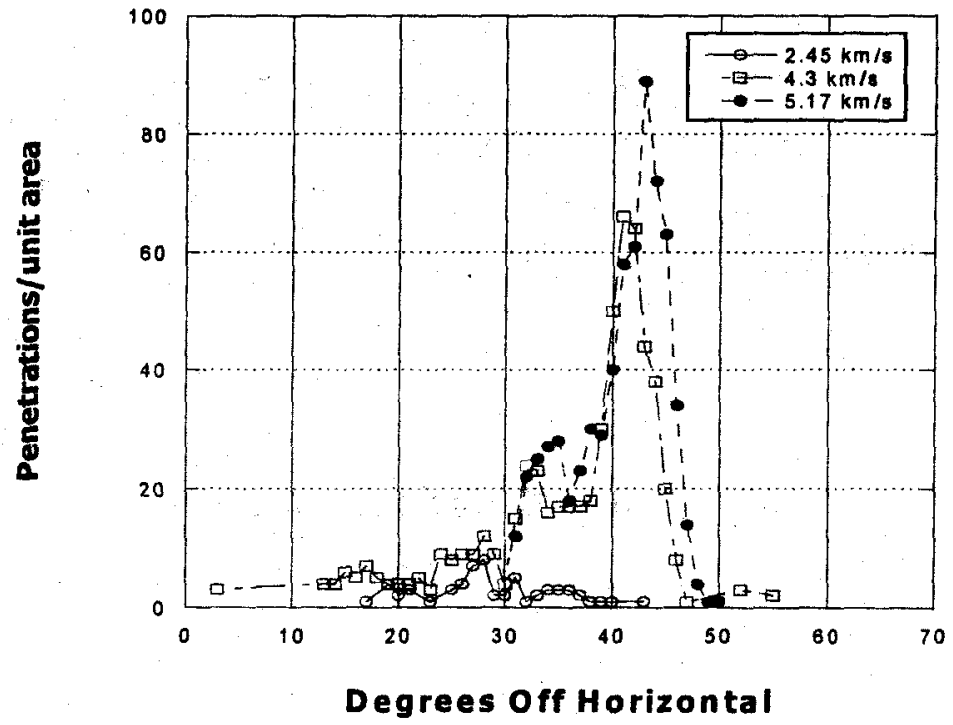

Figure 5. Angular distributions of JSC-1A ejecta with sufficient velocity to penetrate the aluminum foil detectors. 


\section{SUMMARY AND DISCUSSION}

The Ames Vertical Gun Range (AVGR) was used to accelerate spherical Pyrex projectiles of $0.29 \mathrm{~g}$ to velocities ranging between $2.45 \mathrm{~km} / \mathrm{s}$ and $5.18 \mathrm{~km} / \mathrm{s}$. Impact on the powdered pumice and JSC-1A targets occurred at normal incidence. The test series on the pumice targets occurred in September 2006 and the test series on JSC-1A targets occurred approximately 1 year later in August of 2007.

The ejected particles were detected by thin aluminum foil targets placed around the powdered targets in a vacuum chamber maintained at a vacuum level of 0.5 Torr during the impact test. The pumice target was fine grain, $60 \mu \mathrm{m}$ diameter particles and the JSC-1A was a larger grain powdered target with mean grain size of $188 \mu \mathrm{m}$ and a median grain size of $103 \mu \mathrm{m}^{4}$. The results presented in this paper indicate that a peak ejection angle for penetrating ejecta is approximately $38^{\circ}$ off the horizontal for pumice. The peak ejection angle is approximately $42^{\circ}$ to $43^{\circ}$ for JSC-1A.

Previous work by Yamamoto $^{5}$ resulted in a peak ejection angle of approximately $30^{\circ}$. Yamamoto et al. used a "staple-shaped" copper projectile with impact velocities ranging from $243 \mathrm{~m} / \mathrm{s}$ to $272 \mathrm{~m} / \mathrm{s}$ and impacted a target consisting of soda-lime particles with a nominal diameter of $220 \mu \mathrm{m}$. Cintala et $a^{6}$ performed a series of impact tests using spherical aluminum particles accelerated to velocities ranging from 0.8 to $1.92 \mathrm{~km} / \mathrm{s}^{6}$. The incident projectiles had a nominal diameter of $4.76 \mathrm{~mm}$ and impacted coarse-grained sand with grain sizes ranging from $1-3 \mathrm{~mm}$.

Cintala provides extensive detail for characterizing the ejecta angular and size distributions and recorded ejecta angles ranging between $38^{\circ}$ and $55^{\circ}$. Cintala, Yamamoto, and this work used varying techniques to determine ejecta distributions, with Cintala and Yamamoto also providing ejecta velocities.

This experiment made no attempt to measure ejection velocities. Speculation on anticipated ejecta velocities was aided by referencing Yamamoto's work, which states "In the case of the vertical impact of the projectile, most ejecta have velocities lower than $24 \%$ of the projectile speed"5. Table 1 was generated using the assumption that Yamamoto's $24 \%$ prediction can serve as a guide to approximate the peak ejecta velocities.

Table 1. Table showing upper threshold velocities, penetration velocities, and total number of penetrations summed over a hemispherical distribution for pumice and JSC-1A.

\begin{tabular}{|l|r|r|l|l|r|}
\hline & $\begin{array}{l}\text { Incident } \\
\text { velocity } \\
(\mathbf{k m} / \mathbf{s})\end{array}$ & $\begin{array}{l}\text { Ejecta } \\
\text { Upper } \\
\text { Threshold } \\
\text { Velocities } \\
(\mathbf{m} / \mathbf{s})\end{array}$ & $\begin{array}{l}\text { Median } \\
\text { Regolith } \\
\text { particle } \\
\text { diameter } \\
\text { (microns) }\end{array}$ & $\begin{array}{l}\text { lenetration } \\
\text { Threshold } \\
\text { velocity } \\
(\mathbf{m} / \mathbf{s})\end{array}$ & $\begin{array}{l}\text { Total number of } \\
\text { penetrations/hemisphere }\end{array}$ \\
\hline Pumice & 2.5 & 600 & 60 & 988 & 0 \\
\hline Pumice & 3.78 & 907 & 60 & 988 & 259.75 \\
\hline Pumice & 5.18 & 1243 & 60 & 988 & 507.63 \\
\hline JSC1-A & 2.45 & 588 & 188 & 127 & 1397.2 \\
\hline JSC1-A & 4.3 & 1032 & 188 & 127 & 15689.8 \\
\hline JSC1-A & 5.17 & 1240 & 188 & 127 & 18748.8 \\
\hline
\end{tabular}


Using the penetration equation given in NASA SP-8013 (P. 7) describing threshold penetrations of "single thin ductile metal plates", the penetration threshold velocity for the aluminum foil is approximately $988 \mathrm{~m} / \mathrm{s}$ for pumice ejecta and $127 \mathrm{~m} / \mathrm{s}$ for the median particle diameter of the JSC-1 A. This equation is:

$$
t=K_{1} \rho^{1 / 6} m^{0.352} v^{0.875}
$$

In equation $1, t$ is the thickness of the foil penetrated, $K_{1}$ is a constant (.54), $\rho$ is the mass density of the ejecta $\left(1.3 \mathrm{gm} / \mathrm{cm}^{3}\right.$ for pumice), $\left(2.9 \mathrm{~g} / \mathrm{cm}^{3}\right.$ for JSC- $\left.1 \mathrm{~A}\right), \mathrm{m}$ is the mass of the ejecta particle, and $V$ is the ejecta velocity required to penetrate the aluminum foil detector. If Yamamoto's $24 \%$ prediction holds true for this body of work, then there is an explanation as to why the impact in pumice at $2.5 \mathrm{~km} / \mathrm{s}$ did not produce ejecta that penetrated the aluminum foil and why the 3.78 and $5.18 \mathrm{~km} / \mathrm{s}$ impacts in pumice and all the impacts into JSC-1A did produce foil penetrating ejecta. This explanation needs to be confirmed by future experiment.

A numerical summation was performed top determine the rough order of magnitude of total ejecta particles that would be ejected from the target over a hemispherical distribution with sufficient velocity to penetrate the aluminum foil detectors. The number of unit areas, $A_{T}$, was calculated at a distance, $r$, from the impact site. Figure 2 shows the average distance from the foil detector to the impact site as $1.2 \mathrm{~m}$. Actually, the distance from the foil to the impact site was slight different for each foil position. The circumference area at the unique distance, $r$, was calculated. This number was divided by the length of the unit area, $77.42 \mathrm{~cm}$ to determine the number of unit areas per band. Equation 2, below, describes this simplistic approach.

$$
\mathrm{A}_{\mathrm{T}}=(2 \pi \mathrm{r}) / 77.42 \mathrm{~cm}
$$

The total number of ejecta particles with velocity sufficient to penetrate the aluminum foil as a function of degrees off the horizontal is shown in figure 6. The summed total of penetrations over the hemispherical distribution is shown in table 1 .
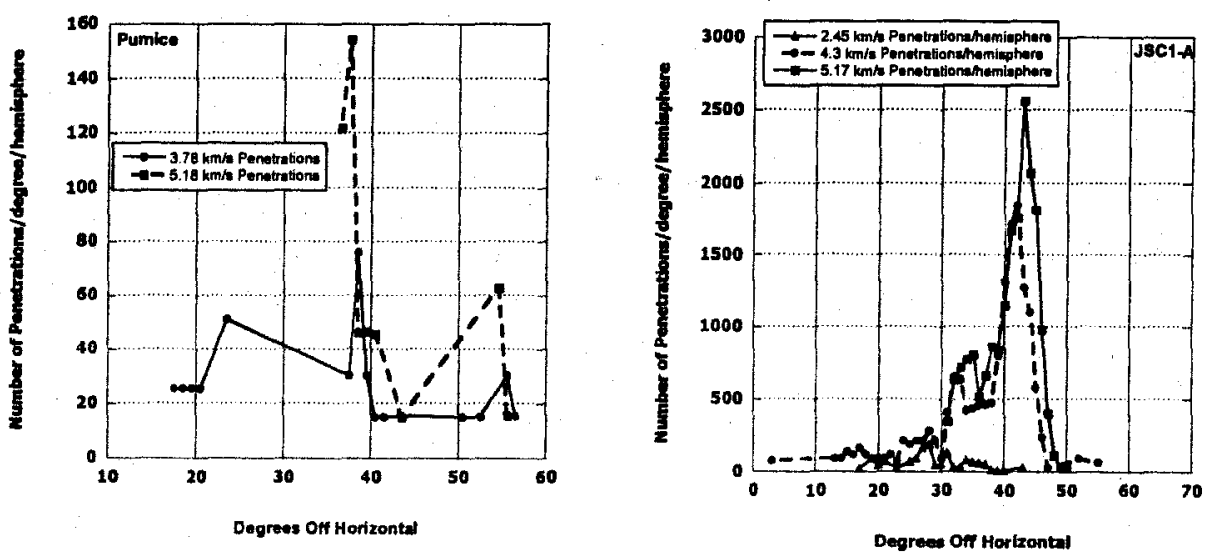

Figure 6. Number of penetrations per degree over a hemispherical distribution for pumice and JSC-1 A targets. 
The lunar meteoroid ejecta environment needs to be better characterized. This work, integrated with other activities being initiated by NASA's Meteoroid Environment Office and the Constellation program, can lead to a more accurate understanding of the ejecta environment. To build on this existing database, additional tests at the AVGR are scheduled. These tests will continue to vary the regolith targets increasing our understanding of the relationship between impact energy, target density, and ejecta distribution.

\section{REFERENCES}

1. Dino J., ed. http://www.nasa.gov/centers/ames/research/technology-onepagers/rangecomplex.html cited 5 May 2007 (2007)

2. NASA SP-8013 Meteoroid Environment Model - 1969 [Near Earth to Lunar Surfacel, March 1969.

3. NASA SP-30425, Space Station Program Natural Environment Definition for Design, Revision B February 8, 1994.

4. Gustafson, Marty www.Junarmarssimulant.com cited 6/28/2007.

5. Yamamoto, S. "Measurement of Impact Ejecta from Regolith Targets in Oblique Impacts", Icarus 158, 87-97 (2002)

6. Cintala M.J., Berthoud, L., Hörz, F., "Ejection-velocity distributions from impacts into coarsegrained sand", Meteoritics \& Planetary Science 34, 605-623 (1999). 


\section{Analysis of Regolith Simulant Ejecta Distributions from Normal Incident Hypervelocity Impact}




\section{Outline}

Analysis of Regolith Simulant Ejecta Distributions from Normal Incident Hypervelocity Impact

- Background

- Discussion of the Problem

- Overview of the AVGR Test System

- Description of the Experiment(s)

- Results

- Summary 


\section{Automated Lunar and Meteor Observatory}

Remotely operated from control building

Recording Devices

Telescopes

2 Meade RCX400 14" Advanced

Ritchey-Chrétien

Astrovid Stellacam

Sony Digital 8 recorder as digitizer

Firewire to PC harddisk
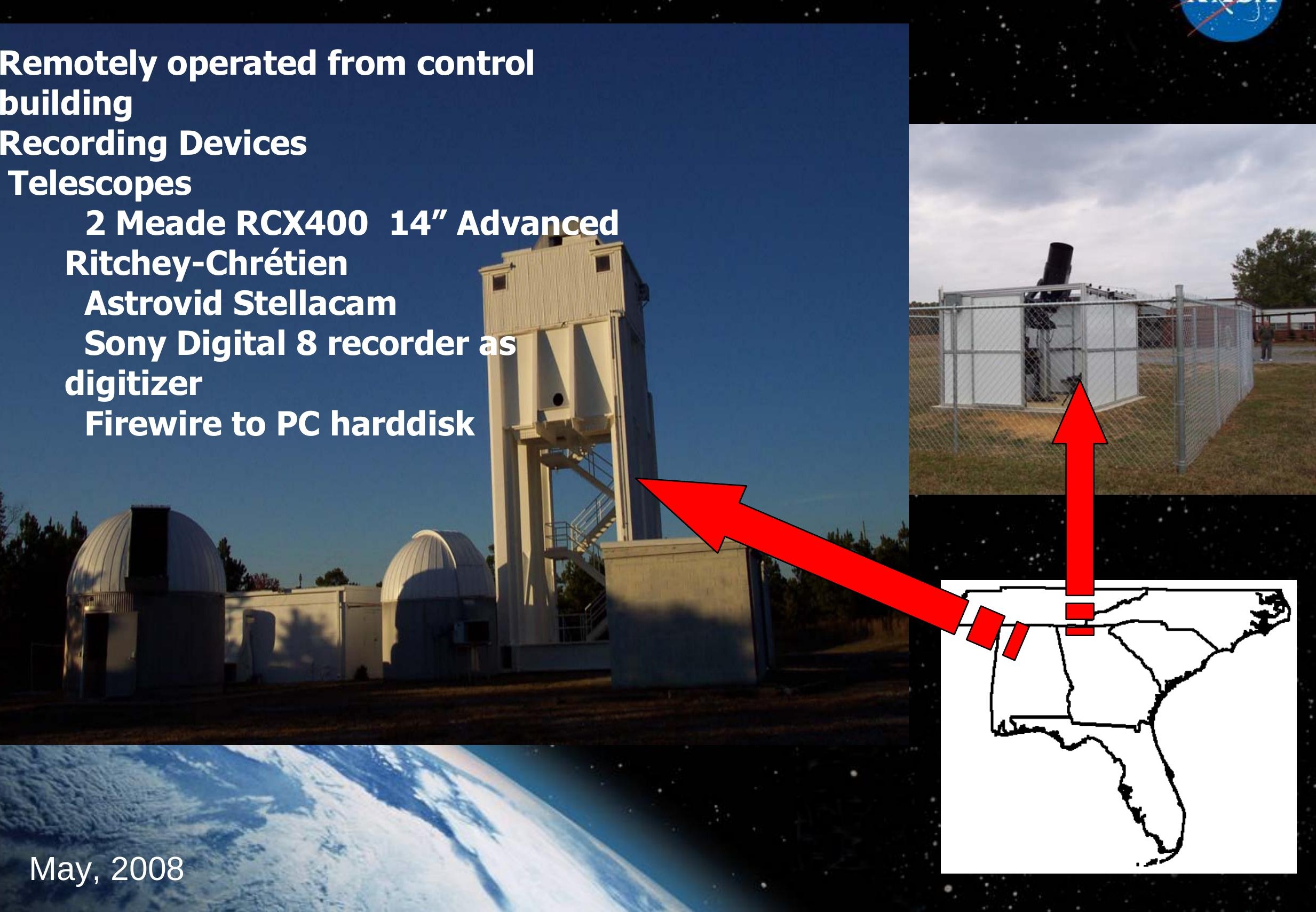


\section{Observing the Moon}

- Dark (not sunlit) side only

- Earthshine illuminates lunar features

- Crescent and quarter phases -0.1 to 0.5 solar illumination

- 5 nights waxing (evening)

- 5 nights waning (morning)

- 4-6 nights of data a month, weather dependent

- Observing procedure

- Aim scopes at Moon

- Record video with WinDV

- $\mathrm{CCD}$ camera $\rightarrow$ Digital 8 recorder $\rightarrow$ hard ${ }^{2}$ ? drive

- Adjust tracking occasionally

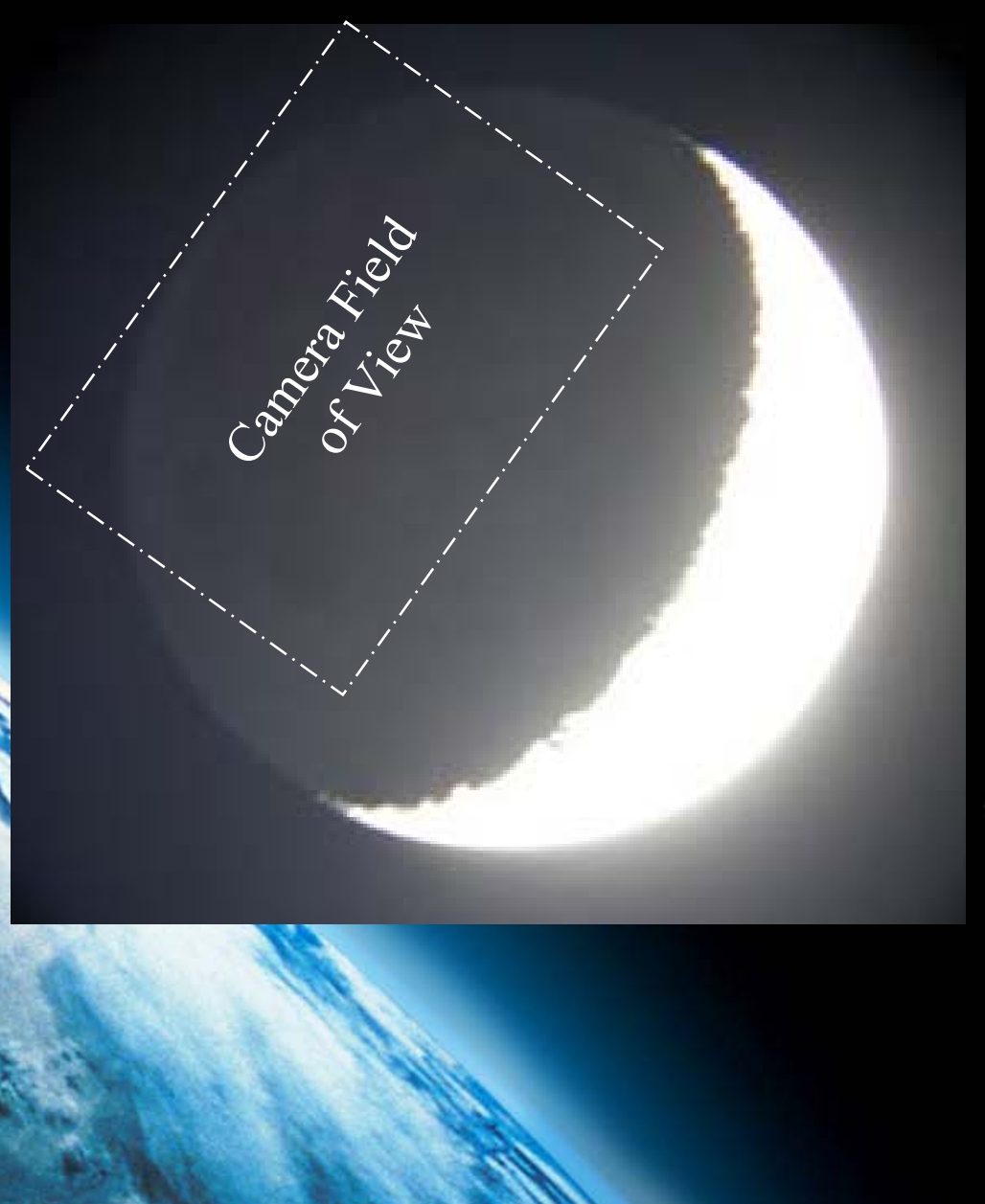


1 



\section{The Problem}

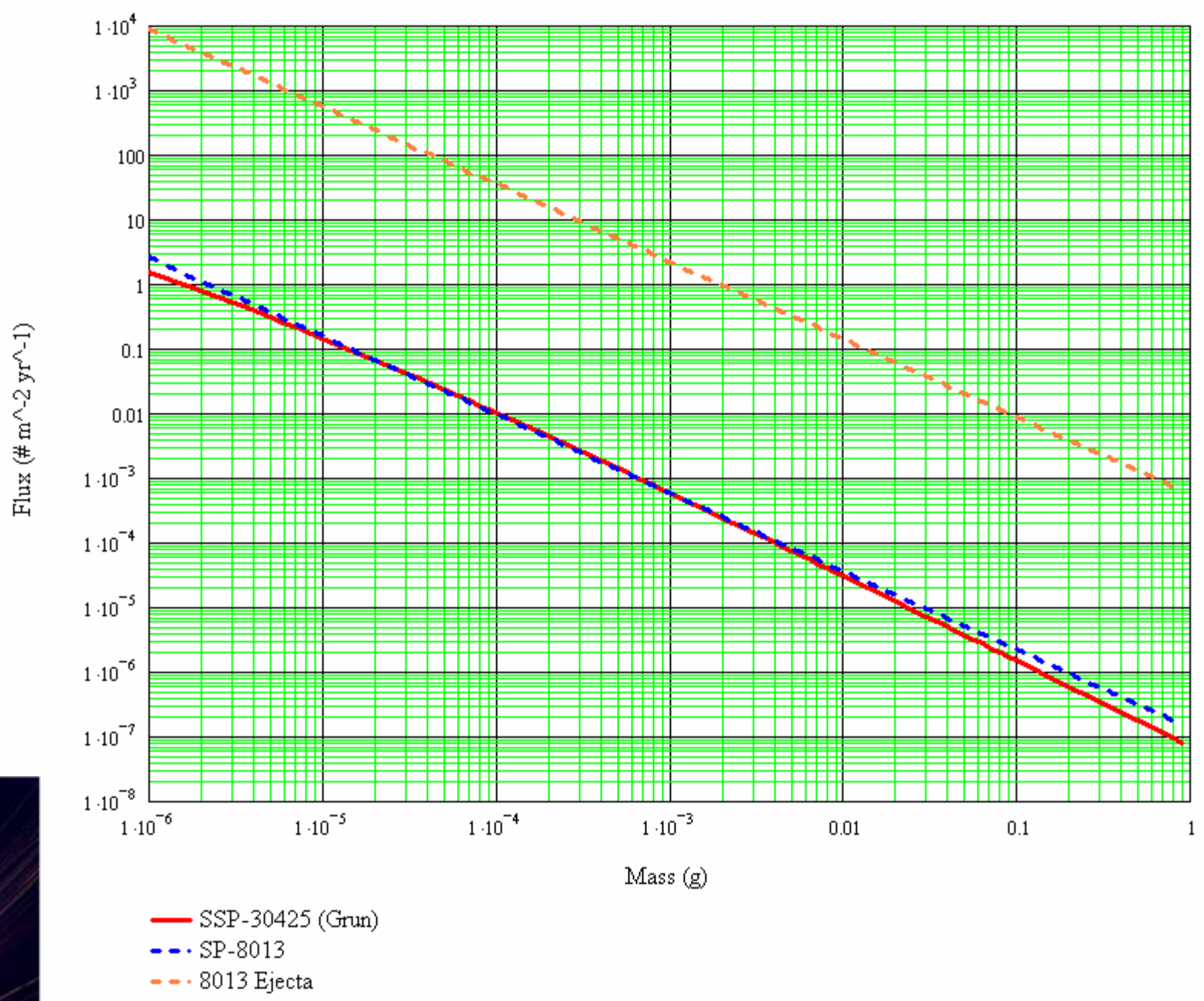




\section{Ames Vertical Gun Range (AVGR)}

0.30 Caliber Light Gas Gun

Projectile velocity range 0.5 to $7 \mathrm{~km} / \mathrm{s}$ Adjustable I mpact Angle $30^{\circ}, 45^{\circ}, 60^{\circ}, 90^{\circ}$

0.5 Torr vacuum in Chamber

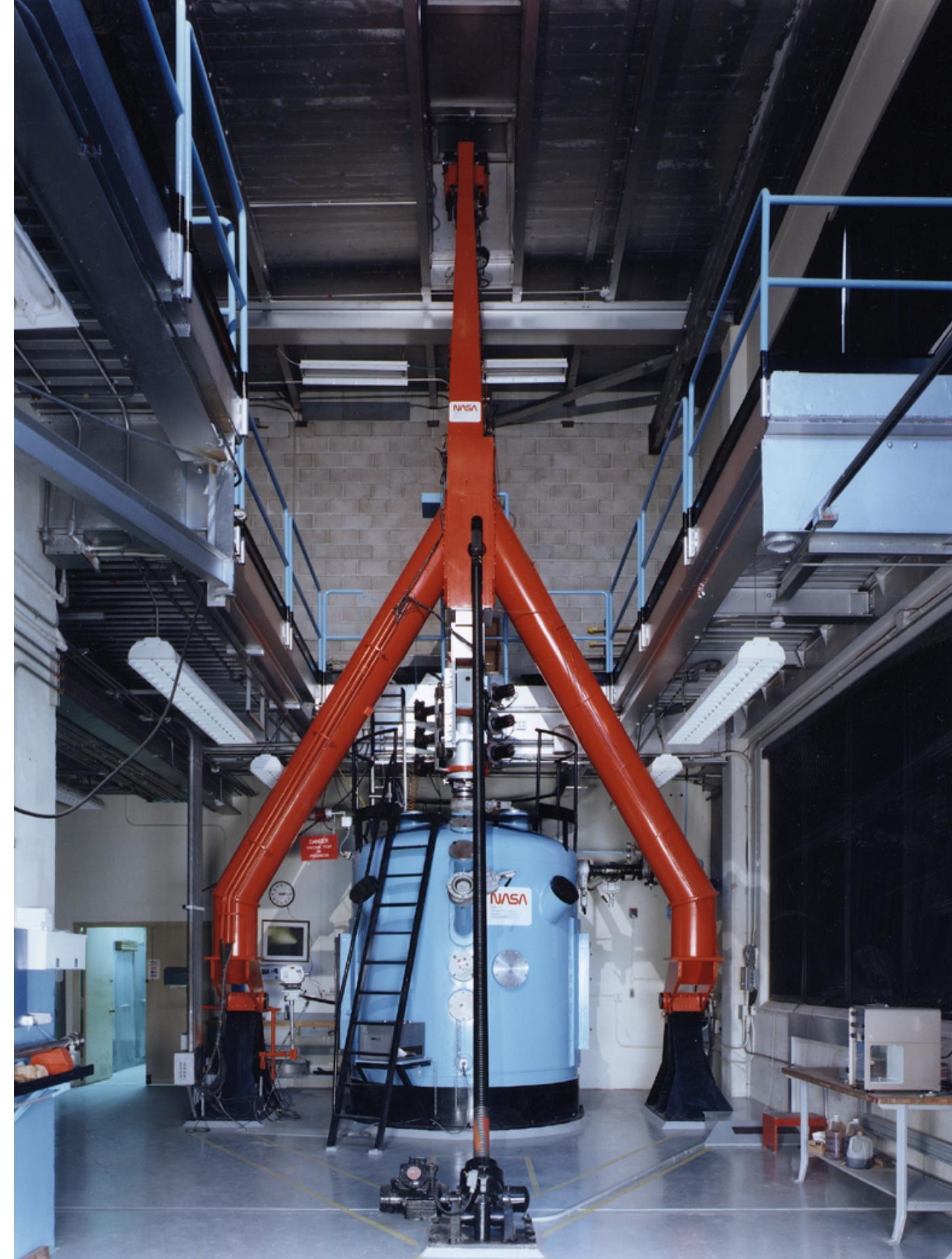


Al Foil Detector positions D \& B for Pumice Test shots AL Foil Detector position C for JSC1-A Test shots
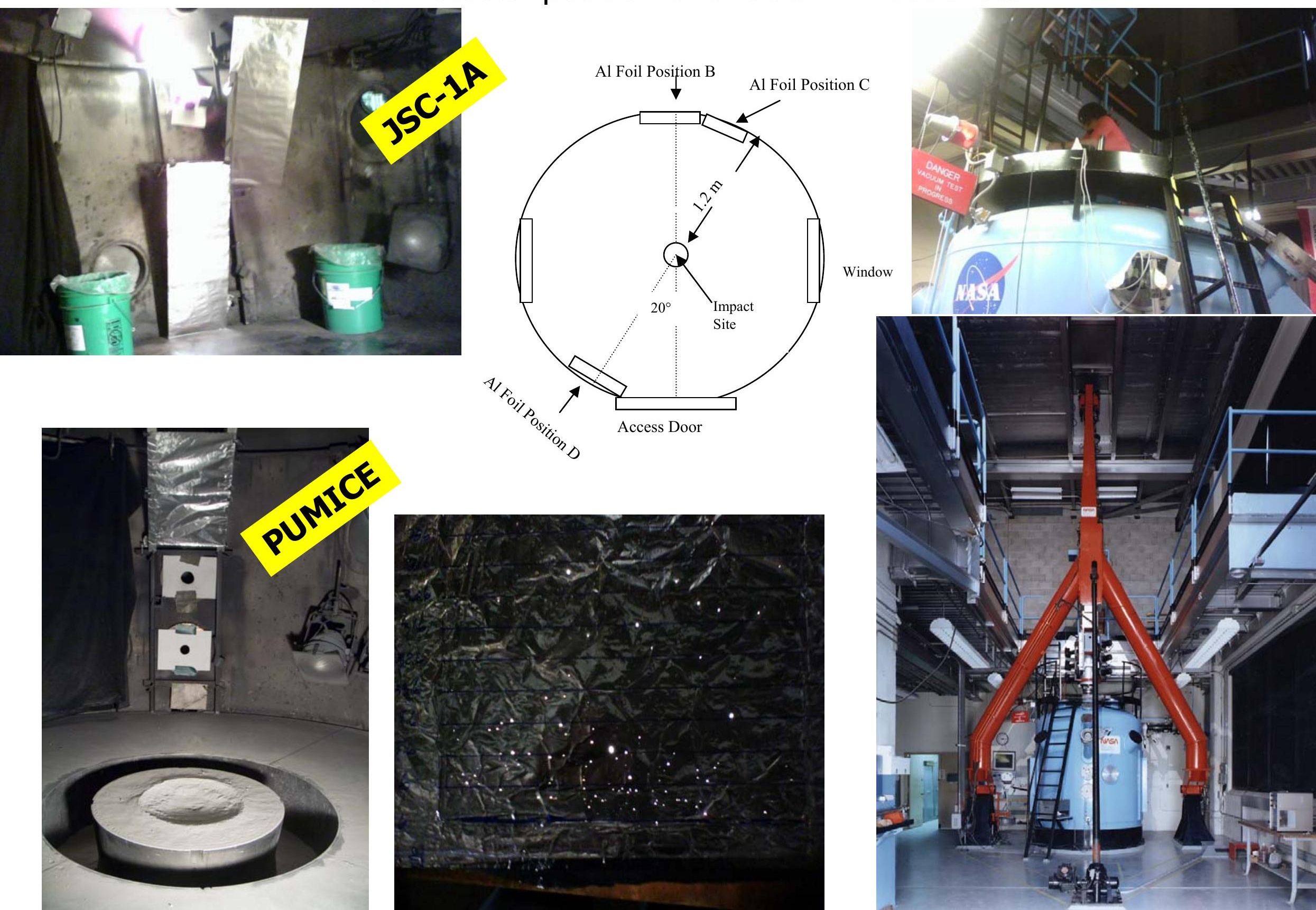


\section{The Results}

\section{JSC-1A}

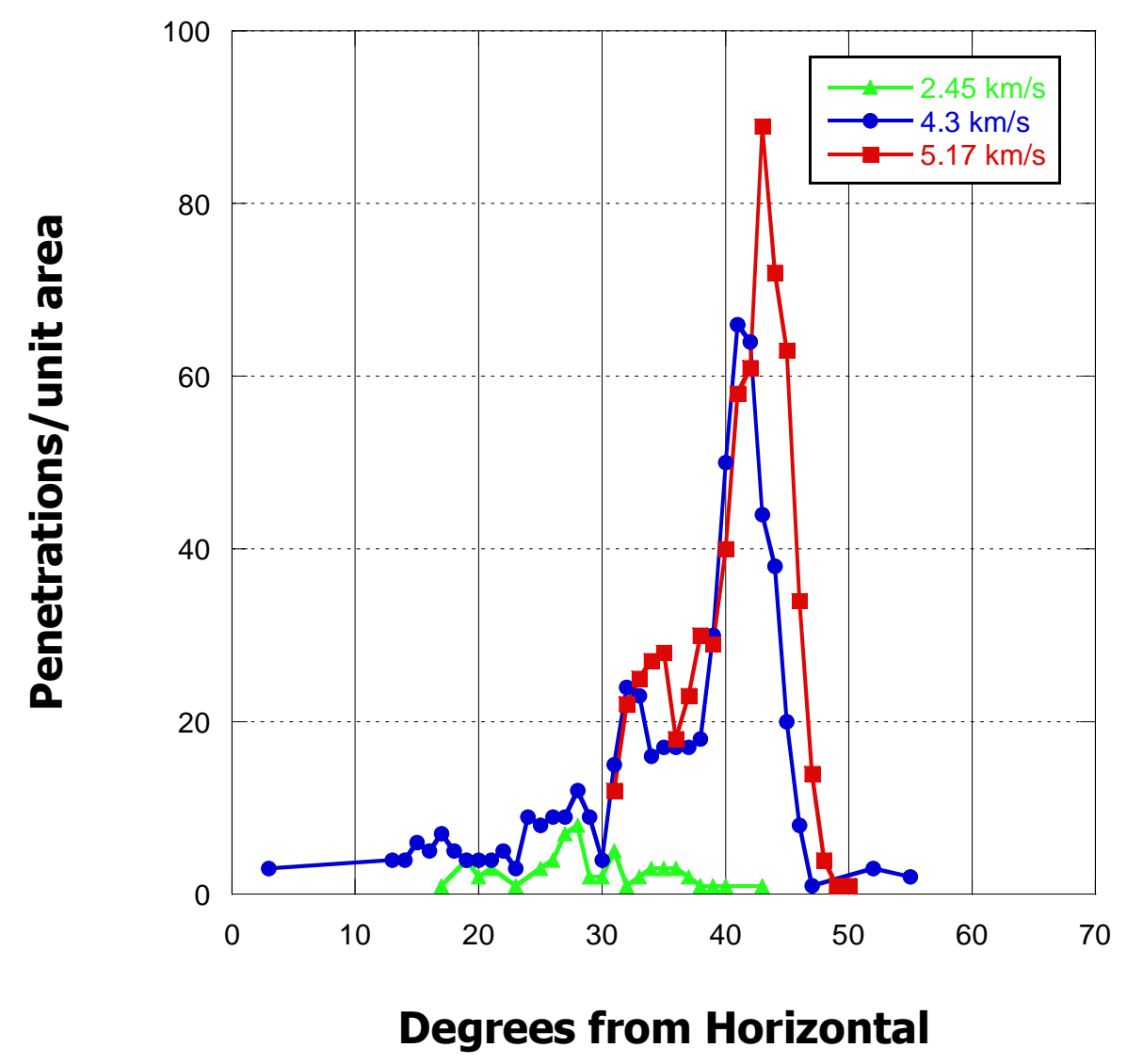

\section{Pumice}

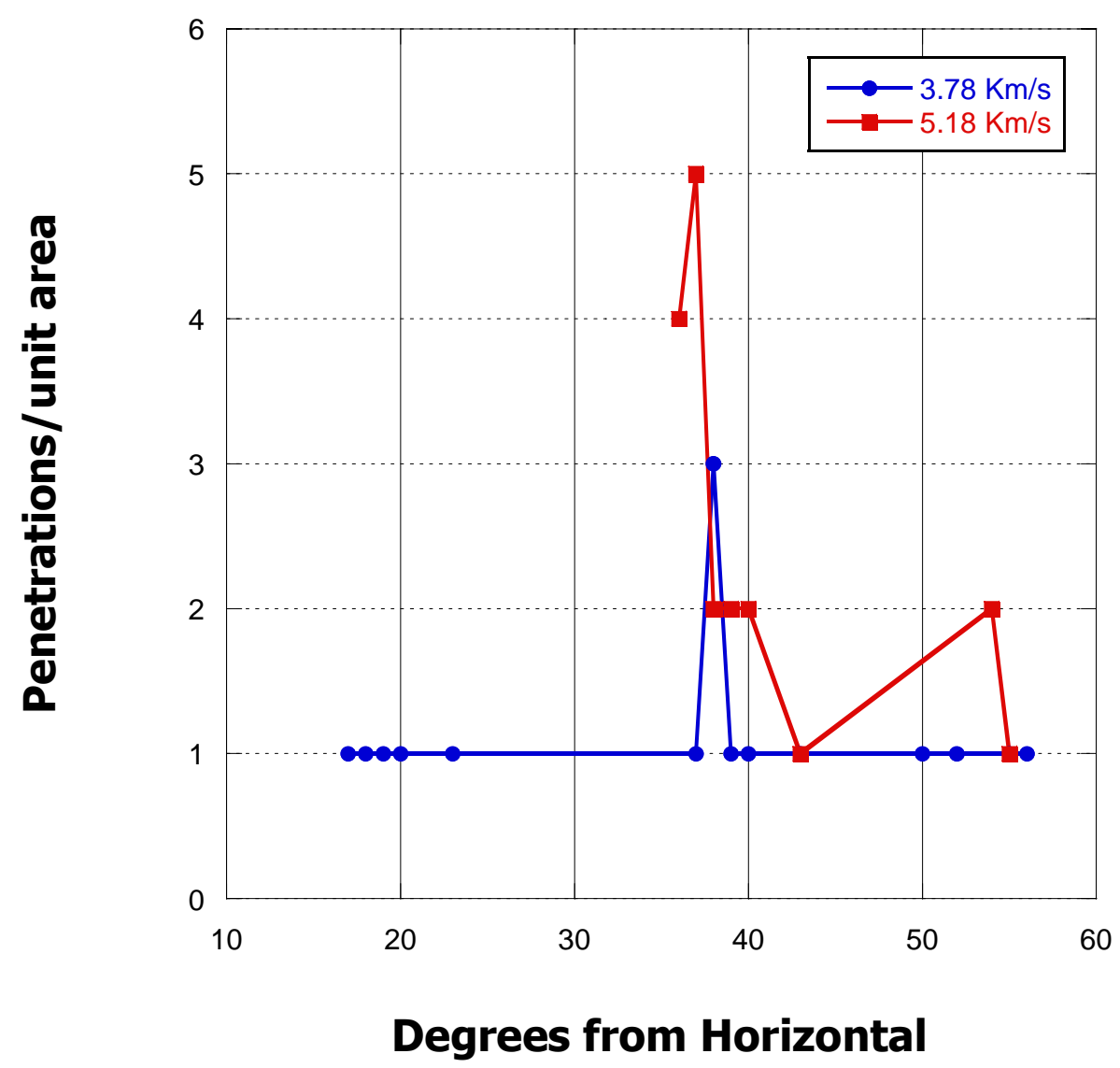




\section{Estimation of Velocity Constraints}

$K_{1}$ is a constant ( 0.54$)$

$$
t=K_{1} \rho^{1 / 6} m^{0.352} V^{0.875}
$$

$\rho$ is the mass density of the ejecta ( $1.3 \mathrm{~g} / \mathrm{cm}^{3}$ for pumice: $2.9 \mathrm{~g} / \mathrm{cm}^{3}$ for JSC-1A)

Mis the mass of the ejecta particle

$\mathbf{V}$ is the ejecta velocity required to penetrate the aluminum foil of thickness $t$

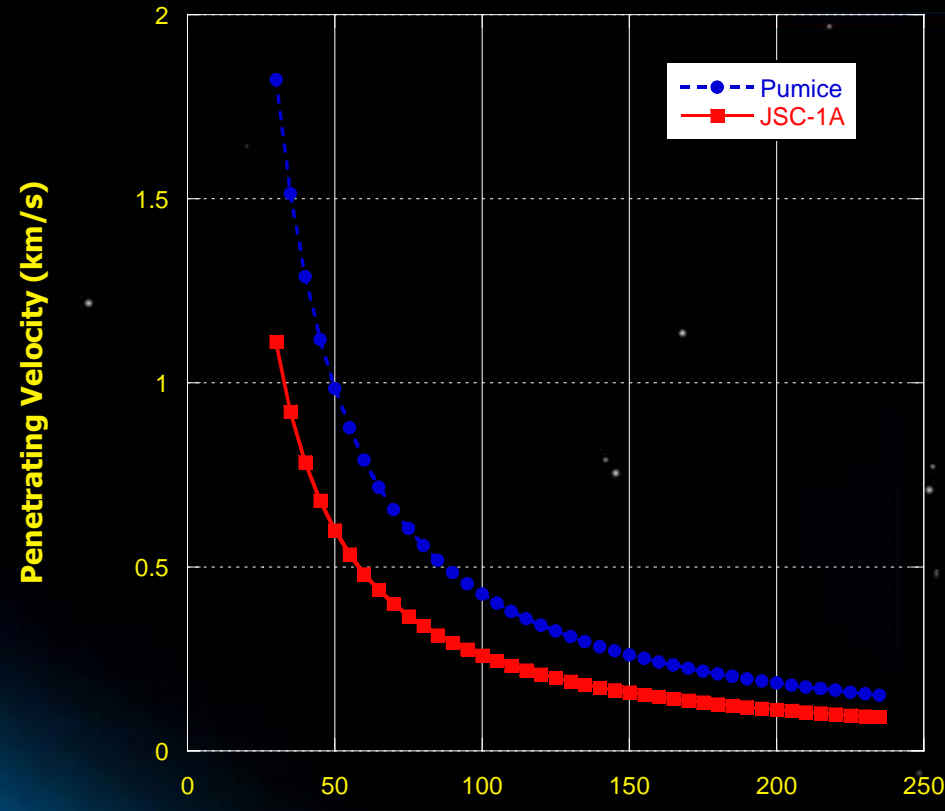

\begin{tabular}{|c|c|c|c|c|c|}
\hline Target & $\begin{array}{l}\text { Incident } \\
\text { velocity } \\
(\mathbf{k m} / \mathbf{s})\end{array}$ & $\begin{array}{l}\text { Ejecta } \\
\text { Upper } \\
\text { Threshold } \\
(\mathrm{m} / \mathrm{s})\end{array}$ & $\begin{array}{l}\text { Median } \\
\text { Regolith } \\
\text { particle } \\
\text { diameter } \\
\text { (microns) }\end{array}$ & $\begin{array}{l}\text { Penetration } \\
\text { Threshold } \\
\text { velocity } \\
(\mathrm{m} / \mathrm{s})\end{array}$ & $\begin{array}{l}\text { Calculated Total } \\
\text { Number of } \\
\text { Penetrations / } \\
\text { Hemisphere }\end{array}$ \\
\hline Pumice & 2.5 & 600 & 60 & 988 & 0 \\
\hline Pumice & 3.78 & 907 & 60 & 988 & 260 \\
\hline Pumice & 5.18 & 1243 & 60 & 988 & 508 \\
\hline JSC1-A & 2.45 & 588 & 103 & 275 & 1397 \\
\hline JSC1-A & 4.3 & 1032 & 103 & 275 & 15690 \\
\hline JSC1-A & 5.17 & 1240 & 103 & 275 & 18749 \\
\hline
\end{tabular}

Diameter ( microns) 
- AVGR used 0.29g Pyrex spheres to impact Regolith simulaint targets

- Pumice, JSC-1A

- Impact Velocities ranged from 2.45 to $5.18 \mathrm{~km} / \mathrm{s}$

- Peak ejecta angles varied with target materiali

- Pumice - $38^{\circ}$

-JSC-1A - $28^{\circ}$ and $42^{\circ}$ to $43^{\circ}$

- More ejecta particle penẹtrations of Al foil dẹtectors were observed from JSC-1A impacts than Pumice.

- Previous work by Cintala and Yamamoto found:

- Cintala found peak ejection angle ranging from $38^{\circ}$ to $55^{\circ}$ - $0.8-1.92 \mathrm{~km} / \mathrm{s}$ spherical Al impacts in coarse-grained sand

- Yamamoto found peak ejection angle of $30^{\circ}$

- Staple-shaped Cu projectiley

.243 to $272 \mathrm{~m} / \mathrm{s}$

- soda-lime-target

Pene $e_{\text {, }}$ ion equations indicate

Rumb jecta penetration threshold is $988 \mathrm{~m} / \mathrm{s}$

$-4-A$ ta penetration threshold is $127 \mathrm{~m} / \mathrm{s}$

Tre ejecta velocity is needed 


\section{Back upp slides}

May, 2008 


\section{Cintala}

Spherical Al particles

0.8 to $1.92 \mathrm{~km} / \mathrm{s}$

$4.76 \mathrm{~mm}$ diameter

Coarse-grained sand

1-3 mm diameter

Peak ejection angle between $38^{\circ}$ and $55^{\circ}$

\section{Yamamoto}

Staple-shaped Cu projectile 243 to $272 \mathrm{~m} / \mathrm{s}$ Soda-lime target 220 um diameter Peak ejection angle $30^{\circ}$ 